\title{
Do patients with a continuous-flow left ventricular assist device benefit from induced-pulsatility or are we just spinning our wheels?
}

\author{
Carlo R. Bartoli, MD, PhD, and Pavan Atluri, MD
}

\author{
From the Division of Cardiovascular Surgery, Hospital of the University of Pennsylvania, Philadelphia, Pa. \\ Disclosures: Authors have nothing to disclose with regard to commercial support. \\ Received for publication July 10, 2015; accepted for publication July 14, 2015; available ahead of print Aug 10, \\ 2015. \\ Address for reprints: Pavan Atluri, MD, Division of Cardiovascular Surgery, University of Pennsylvania, 3400 \\ Spruce St, 6 Silverstein Pavilion, Philadelphia, PA 19140 (E-mail: pavan.atluri@uphs.upenn.edu). \\ J Thorac Cardiovasc Surg 2015;150:945-6 \\ $0022-5223 / \$ 36.00$ \\ Copyright (C) 2015 by The American Association for Thoracic Surgery \\ http://dx.doi.org/10.1016/j.jtcvs.2015.07.042
}

The management of continuous-flow left ventricular assist devices (LVADs) continues to evolve. Benefits of pulsatile versus nonpulsatile mechanical circulatory support remain an ongoing debate. Continuous-flow LVADs have demonstrated improved outcomes that had not been possible with prior pulsatile devices. As a result, research and development has focused on smaller, more durable continuousflow devices as a means of improving patient outcomes. Continuos-flow devices have introduced new challenges. Specifically, the optimal speed of the pump, degree of left ventricular unloading, status of the aortic valve, and level of native pulsatility remain unknown.

In recent years, it has become common practice to reduce the speed of continuous-flow LVADs to promote aortic valve opening and pulsatility. Potential benefits of pulsatility include the prevention of aortic valve thrombosis, leaflet fusion, and de novo aortic insufficiency, reduced arterial stiffening, better end-organ function, and possibly reduced gastrointestinal bleeding. Indeed, a popular management strategy for LVAD-associated bleeding, which occurs in $40 \%$ to $75 \%$ of patients with LVADs, is to reduce LVAD speed (along with anticoagulation and antiplatelet therapy). However, there is emerging evidence that lower LVAD speed itself may not reduce bleeding and may actually promote thrombosis. $^{1,2}$

In this issue of the Journal, Yang and colleagues ${ }^{2}$ demonstrated in a mock circulatory system that reduced continuous-flow LVAD speed (with induced pulsatility) caused turbulent flow channels within the pump. ${ }^{2}$ At high LVAD speeds without pulsatility, laminar flow predominated. However, as LVAD speed decreased, pulsatile flow generated chaotic eddies with local recirculation near the proximal LVAD bearing during end diastole. The authors speculated that these turbulent flow channels with local stasis may contribute to thrombosis. They suggested that the recent trend in managing patients with LVADs with lower pump speeds may have contributed to the recent abrupt increase in LVAD thrombosis. $^{3,4}$

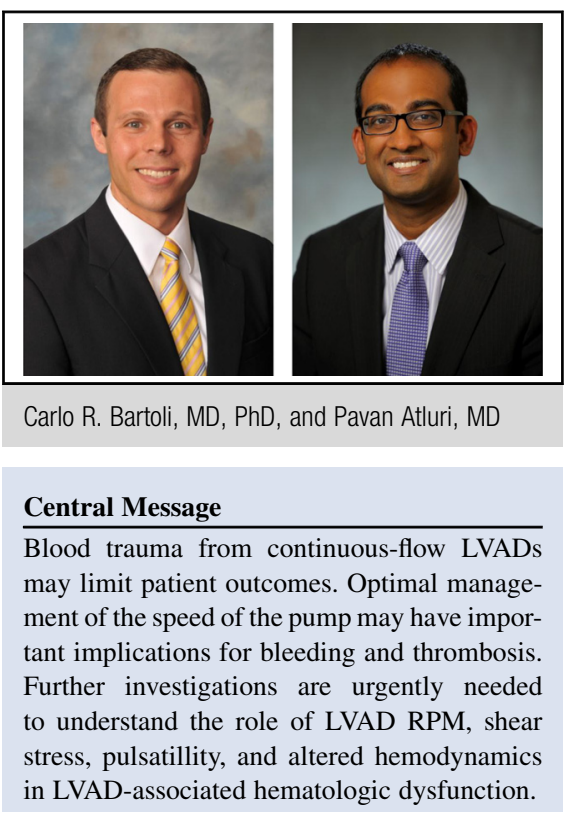

See Article page 938.

The underlying causes of LVAD thrombosis remain unclear, especially in patients with therapeutic anticoagulation. Multiple mechanisms predispose to clot formation. ${ }^{5}$ Prolonged blood contact with the artificial interior surface of the LVAD activates platelets, leukocytes, the complement system, and the coagulation cascade. Activation of endothelial cells and generation of microparticles during LVAD support also may contribute to hypercoagulability. However, recent evidence suggests that abnormal blood flow from continuous-flow LVADs plays a major role. Current continuous-flow LVADs generate nonphysiologic hemodynamics $^{6}$ with abnormal pulsatility, turbulence at the end-to-side outflow graft anastomosis, and shear stress 1 to 2 orders of magnitude greater than physiologic values. ${ }^{7}$ Importantly, shear stress is a potent activator of thrombogenesis. 8

Many of the factors that predispose to LVAD thrombosis also may impair primary hemostasis and produce a bleeding diathesis with similarities to von Willebrand syndrome (type II). Supraphysiologic shear stress from the LVAD accelerates von Willebrand factor (vWF) metabolism and depletes functional vWF multimers. ${ }^{9}$ The acquired vWF deficiency predisposes patients with LVADs to nonsurgical bleeding. The clinical scenario becomes especially challenging when a patient with an LVAD who is anticoagulated 
presents with bleeding. In addition to reducing anticoagulation and discontinuing antiplatelet therapy, it has been proposed that lower LVAD speeds (with induced pulsatility) decrease shear stress and may thereby reduce vWF degradation and bleeding. ${ }^{10}$ And there is evidence that pulsatility protects against LVAD-associated bleeding. ${ }^{10}$ However, a recent experimental study demonstrated that LVAD speeds within the clinical operational range did not reduce shear stress to within physiologic levels, and reduced LVAD speed, itself, did not reduce vWF degradation. This finding and the recent findings reported by Yang and colleagues ${ }^{2}$ suggest that lower LVAD speed may not be an appropriate management strategy for bleeding and may inadvertently predispose patients to thrombosis. It will be interesting to see whether these and other problems attributed to continuous flow will be minimized with newer-generation continuous-flow LVADs that incorporate pulsatility algorithms to generate pulsatile flow.

The study by Yang and colleagues ${ }^{2}$ highlights the importance of hemorheology in LVAD-associated hematologic (dys)function. As we learn more about the interface between blood and the nonphysiologic hemodynamics of an LVAD, patient management strategies will continue to mature. Yang and colleagues ${ }^{2}$ should be commended for their important work, which suggests that reduced LVAD speeds may promote LVAD thrombosis. It will be critical for these findings to be validated in large animal models and clinical studies before changing patient management. Indeed, further investigation into hemodynamic contributions to bleeding and thrombosis are urgently needed in order to optimize LVAD therapy and improve outcomes. We thank the Editors for the opportunity to comment on an important and evolving topic in the field of mechanical circulatory support.

\section{References}

1. Wong K, Samaroo G, Ling I, Dembitsky W, Adamson R, del Álamo JC, et al. Intraventricular flow patterns and stasis in the LVAD-assisted heart. J Biomech. 2014; 47:1485-94.

2. Yang F, Kormos RL, Antaki JF. High-speed visualization of disturbed pathlines in axial flow ventricular assist device under pulsatile conditions. J Thorac Cardiovasc Surg. 2015;150:938-44.

3. Starling RC, Moazami N, Silvestry SC, Ewald G, Rogers JG, Milano CA, et al. Unexpected abrupt increase in left ventricular assist device thrombosis. $N$ Engl J Med. 2014;370:33-40.

4. Kirklin JK, Naftel DC, Kormos RL, Pagani FD, Myers SL, Stevenson LW, et al. Interagency Registry for Mechanically Assisted Circulatory Support (INTERMACS) analysis of pump thrombosis in the HeartMate II left ventricular assist device. J Heart Lung Transplant. 2014;33:12-22.

5. Bartoli CR, Ailawadi G, Kern JA. Diagnosis, nonsurgical management, and prevention of LVAD thrombosis. J Card Surg. 2014;29:83-94.

6. Bartoli CR, Giridharan GA, Litwak KN, Sobieski M, Prabhu SD, Slaughter MS, et al. Hemodynamic responses to continuous versus pulsatile mechanical unloading of the failing left ventricle. ASAIO J. 2010;56:410-6.

7. Fraser KH, Zhang T, Taskin ME, Griffith BP, Wu ZJ. A quantitative comparison of mechanical blood damage parameters in rotary ventricular assist devices: shear stress, exposure time and hemolysis index. J Biomech Eng. 2012;134:081002.

8. Nesbitt WS, Westein E, Tovar-Lopez FJ, Tolouei E, Mitchell A, Fu J, et al. A shear gradient-dependent platelet aggregation mechanism drives thrombus formation. Nat Med. 2009;15:665-73.

9. Bartoli CR, Restle DJ, Zhang DM, Acker MA, Atluri P. Pathologic von Willebrand factor degradation with a left ventricular assist device occurs via two distinct mechanisms: mechanical demolition and enzymatic cleavage. J Thorac Cardiovasc Surg. 2015;149:281-9.

10. Wever-Pinzon O, Selzman CH, Drakos SG, Saidi A, Stoddard GJ, Gilbert EM, et al. Pulsatility and the risk of nonsurgical bleeding in patients supported with the continuous-flow left ventricular assist device HeartMate II. Circ Heart Fail. 2013;6:517-26. 